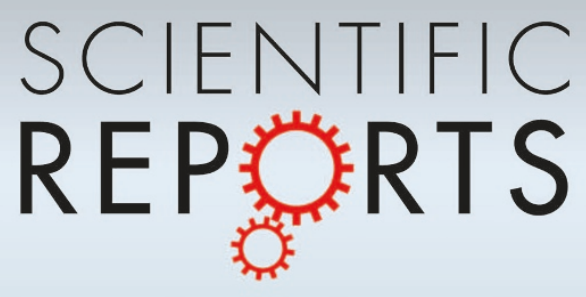

\title{
OPEN Magnetic Resonance Imaging of Electrolysis.
}

SUBJECT AREAS:

MAGNETIC RESONANCE

IMAGING

BIOMEDICAL ENGINEERING

Received

14 July 2014

Accepted

6 January 2015

Published

9 February 2015

Correspondence and requests for materials should be addressed to

M.H. (mhjouj@

hotmail.com; mhjouj@

hpro.alquds.edu)

* These authors contributed equally to this work.

\author{
Arie Meir ${ }^{1 *}$, Mohammad Hjouj ${ }^{2 *}$, Liel Rubinsky ${ }^{3}$ \& Boris Rubinsky ${ }^{1,3}$
}

${ }^{1}$ Graduate Program in Biophysics, University of California Berkeley, Berkeley, CA 94720, ${ }^{2}$ Medical Imaging Department; Faculty of Health Professions, Al-Quds University/Abu Dies/Jerusalem, ${ }^{3}$ Department of Mechanical Engineering, University of California Berkeley, Berkeley, CA 94720.

This study explores the hypothesis that Magnetic Resonance Imaging (MRI) can image the process of electrolysis by detecting $\mathrm{pH}$ fronts. The study has relevance to real time control of cell ablation with electrolysis. To investigate the hypothesis we compare the following MR imaging sequences: T1 weighted, T2 weighted and Proton Density (PD), with optical images acquired using $\mathrm{pH}$-sensitive dyes embedded in a physiological saline agar solution phantom treated with electrolysis and discrete measurements with a $\mathrm{pH}$ microprobe. We further demonstrate the biological relevance of our work using a bacterial $E$. Coli model, grown on the phantom. The results demonstrate the ability of MRI to image electrolysis produced $\mathrm{pH}$ changes in a physiological saline phantom and show that these changes correlate with cell death in the E. Coli model grown on the phantom. The results are promising and invite further experimental research.

$\mathrm{n}$ a biomedical context, electrolysis refers to the passage of low magnitude, direct current between two electrodes through a living tissue. Electrolytic ablation is the application of electrolysis for inducing irreversible damage to undesired tissues, and research into the biochemical/biophysical aspects of tissue electrolysis can be traced to the $19^{\text {th }}$ century ${ }^{1}$. In modern medicine, the tools available for tissue ablation are based on various biophysical and biochemical processes. Thermally based approaches include heating, cooling or freezing, while electroporation, injection of chemical agents, sonoporation and photodynamic effects are additional examples for more recent developments which were suggested as ways to expand the surgical arsenal. Electrolysis is a part of this toolkit and the basic underlying mechanism revolves around the ionic species in the tissue, species, which change into compounds that can ablate cells.

A large body of the modern electrolysis research can be traced back to the work of Nordenstrom and colleagues $^{2,3}$. To understand the effects of electrolysis on tissue, researchers apply tools like histology and mathematical models of the involved electrochemical processes ${ }^{4-14}$. Accompanying the more fundamental research efforts, there is a growing body of clinical work, e.g. ${ }^{15-18}$. This work was inspired by several findings and the development of a few research techniques: it was shown that the electrolysis induced $\mathrm{pH}$ changes can be used to reliably monitor the extent of tissue ablation ${ }^{19}$. These findings have led to several basic studies on quantifying the process of electrolysis through the use of transparent gels with $\mathrm{pH}$ dyes ${ }^{11,20,21}$. Expanding the view beyond electrolysis-driven $\mathrm{pH}$ changes, different approaches have been developed to allow monitoring of $\mathrm{pH}$ levels. These include classical instruments such as the glass electrode $e^{22}$, electrochemically based tools such as cyclic voltammetry ${ }^{23,24}$ or semiconductor based micro-electrode $\mathrm{pH}$ sensors ${ }^{25}$. These discrete $\mathrm{pH}$-measuring devices typically have very good $\mathrm{pH}$ resolution. However, they generate discrete data and cannot provide a continuous, spatial image of the $\mathrm{pH}$ distribution. The devices are often sensitive, require recalibration and their response time, even in stirred solutions, is between 5 and 15 seconds. Furthermore, even though the probe tips can be of microscopic size (on the order of microns), the outer diameter of the device's body is on the order of a centimeters, which limits the spatial resolution. To summarize, $\mathrm{pH}$ meters are excellent devices for measuring local $\mathrm{pH}$, when the $\mathrm{pH}$ does not change in time. However, in electrolytic ablation the continuous generation of electrolytic products and their diffusion over time through the treated volume leads to changes in local $\mathrm{pH}$ in both time and space. This requires developing a $\mathrm{pH}$ detection system that can provide a continuous temporal and spatial distribution of the $\mathrm{pH}$ changes. Therefore, while $\mathrm{pH}$-measuring devices can generate excellent discrete local data, they cannot be used for continuous measurements in time (because of the long response time, tissue fouling issues and the need to recalibrate) or in space (because of their discrete nature and size) of the process of electrolysis. The goal of this study is to introduce a technology than can detect changes in $\mathrm{pH}$ in both time and space, to be used as a tool in the context of electrolytic treatment of tissues. 
Table $1 \mid$ MRI parameters used in the study

\begin{tabular}{llrrrrrrr} 
Sequence & \multicolumn{1}{c}{ Coil } & TR $[\mathrm{ms}]$ & TE $[\mathrm{ms}]$ & FOV $[\mathrm{mm}]$ & Slice thickness & Num. slices & \# of excitations NSA & Recon. Matrix size \\
\hline T1W TSE & SENSE-Pediatric Head coil & 500 & 17 & 120 & $2 \mathrm{~mm}$ & 4 & 2 \\
T2W TSE & SENSE-PED-HEAD & 1000 & 100 & 120 & $2 \mathrm{~mm}$ & 4 & 512 \\
PD & SENSE-PED-HEAD & 5000 & 30 & 120 & $2 \mathrm{~mm}$ & 4 & 2 & 2 \\
\hline
\end{tabular}

Medical imaging has been one of the key innovation drivers in the minimally invasive surgery field. Minimally invasive procedures are now part of a common practice for ablating tissue deep inside the body, but most of these procedures cannot be performed without medical imaging ${ }^{26}$. Electrolysis is currently limited by the lack of an effective means to monitor the extent of tissue ablation deep in the body. During electrolysis, $\mathrm{pH}$ fronts caused by evolution of protons $\left(\mathrm{H}^{+}\right)$and hydroxide $\left(\mathrm{OH}^{-}\right)$ions at the electrodes diffuse from the electrodes outward ${ }^{5,27}$. Fundamental studies on tissue ablation by electrolysis have shown that $\mathrm{pH}$ changes are indicative of electrolytic tissue ablation ${ }^{19}$. It was found that $\mathrm{pH}$ dyes marked gels ${ }^{27-29}$, could be used to study, monitor and image electrolysis.

Our work draws from a 1957 paper by Meiboom, Luz and Gill who studied proton relaxation times in water as a function of $\mathrm{pH}^{30}$. During the last several decades, Magnetic Resonance Imaging (MRI) was used to study $\mathrm{pH}$ changes in many bio-medical settings with various methods and for various applications. For example, the effect of intracellular $\mathrm{pH}$, as well as blood and tissue oxygen tension on $\mathrm{T} 1$ relaxation in the rat brain were studied in Ref. 31. Measurements of $\mathrm{pH}$ changes due to ischemia in the brain, in relation to amine and amide protons were reported in Refs 32, 33. Measurements of $\mathrm{pH}$ changes due to kidney failure with an MRI-CEST $\mathrm{pH}$ responsive contrast agent, Iopamidol were presented in Ref. 34. An evaluation of a range of MRI-active $\mathrm{pH}$ indicators for food applications is found in Refs 35, 36. In Ref. 37, it was shown that calf muscle T2 changes correlate with $\mathrm{pH}, \mathrm{PCr}$ recovery and oxidative phosphorylation. Most relevant to this study is the work of Schilling et al, who have found that changes in intracellular $\mathrm{pH}$, affect the relaxation time of $\mathrm{T} 2$ in brain tissue ${ }^{38}$. While an abundance of papers exist on the use of MRI for detecting changes in $\mathrm{pH}$, no publication on the use of MRI for monitoring the development of $\mathrm{pH}$ fronts during electrolysis was found by our literature search. The primary goal of this study is to explore the hypothesis that $\mathrm{pH}$ fronts, produced by electrolysis, can be detected with MRI, for possible application in monitoring and controlling cell ablation with electrolysis. As briefly illustrated by the above literature citations, there are many methods that could be used for detecting $\mathrm{pH}$ changes in tissue with MRI. Inspired by references $^{30,38}$, we chose to explore our hypothesis with basic T1 weighted and $\mathrm{T} 2$ weighted based sequences for water.

To explore the hypothesis we conduct an experimental study using a $\mathrm{pH}$ dye stained physiological saline agar-gel based phantom as a model for a living tissue, from an electrochemical standpoint. The $\mathrm{pH}$ dye generates a continuous temporal and spatial image of the effected region, but is restricted to use in transparent systems. In addition we have collected discrete measurements using a $\mathrm{pH}$ probe - with the limitations of these measurements in mind. In the study we compare images obtained with MRI to optical images acquired using $\mathrm{pH}$-sensitive dyes and the discrete $\mathrm{pH}$ data. The MRI imaging sequences used were $\mathrm{T} 1$ weighted (T1W), T2 weighted (T2W) and Proton Density (PD). The optical images were acquired using $\mathrm{pH}$ sensitive dyes embedded in the agar phantom that is exposed to electrolysis. In addition to validating the MRI-based approach using $\mathrm{pH}$-sensitive dyes, we demonstrate a biological application of our MRI work by comparing a spatial map of bacterial viability exposed to electrolysis with the MRI image of the phantom during electrolytic treatment. Our results are promising, and invite further experimental explorations.

\section{Results}

MRI Experiment Results. As described in our methods, the agar plates were scanned before the administration of electrolytic treatment with the following sequences: T1W, T2W and PD. Then the gel plates were electrolytically treated for 15 minutes using three different voltages: $3 \mathrm{~V}, 6 \mathrm{~V}$, and $9 \mathrm{~V}$. After administering the treatment, the plates were immediately positioned in a pediatric head coil, and inserted into the MR scanner. MR sequences with the same pre-treatment parameters were then acquired. The MRI parameters are presented in Table 1. To facilitate comparison of results, figure 1 brings together images obtained for the three voltages and the three MRI sequences. The three columns are for the voltages of $3 \mathrm{~V}, 6 \mathrm{~V}$ and $9 \mathrm{~V}$, from left to right, respectively. The rows from top to bottom are for the following sequences: T1W, T2W, and PD, respectively. All the images are for a standard Petri dish with the same diameter, $8.5 \mathrm{~cm}$. The electrolysis was administered via the same device, positioned at the same place for all the experiments, as constrained by the application rig in figure $2 \mathrm{a}$. The position of the electrodes can be seen in some images as the two black traces (void of signal) at the centerline of the Petri dish. The distance between the electrodes was $2 \mathrm{~cm}$. In all the images the anode is on the left and the cathode is on the right.

The first row of figure 1 shows images taken with the T1W sequence. The signal from the treated volume is isointense to hypointense. It is isointense for the lower voltage of $3 \mathrm{~V}$ and becomes slightly hypointense with the increasing applied voltage. The $\mathrm{pH}$ change front is barely distinguishable for the $9 \mathrm{~V}$ treatment. The second row shows results obtained with the T2W sequence. The margin of the electrolysis-affected region is marked with dotted yellow line. Hypointense signal can be seen in the treated region, with the signal intensity decreasing with increasing voltage. The affected region near the anode is larger than near the cathode. The altered $\mathrm{pH}$ front appears diffused in the anode-affected region and well-delineated in the cathode affected region. The interface between the cathode affected region and the anode affected region is distinct and visible. It is also note-worthy that in the cathode-affected region, the intensity adjacent to the cathode decreases with increasing voltage. The images produced with PD sequences, presented in the third row, show a generally similar pattern to that described for the T2W sequence produced images. Images produced with the PD sequence show a hypo intense signal with lower intensity relative to the T2W sequence produced images.

pH Dye Experiment. For the $\mathrm{pH}$ dye experiments we have infused the agar gel phantom described in our methods with two $\mathrm{pH}$ sensitive indicator dyes. Figure 3 shows results obtained from the $\mathrm{pH}$ dye experiments. To facilitate the comparison of results, Figure 3 brings together images obtained for the three voltages and results from the two-pH dyes infused gels. The three columns are for voltages of $3 \mathrm{~V}, 6 \mathrm{~V}$ and $9 \mathrm{~V}$, from left to right, respectively. The first row shows results obtained with phenolphthalein staining. The phenolphthalein stain produces a distinct pink color in the $\mathrm{pH}$ range from 8.2 to 12. The first row shows, as expected, an impression in only the cathode region on the right. The margins of the marked regions indicate a minimal $\mathrm{pH}$ of 8.2. For a voltage of $3 \mathrm{~V}$, the change of $\mathrm{pH}$ front takes a circular shape, most likely of a $\mathrm{pH}$ of 8.2. Increasing the voltage increases the size of the change in $\mathrm{pH}$ affected area. Similar to the MRI images, the cathode-affected front 

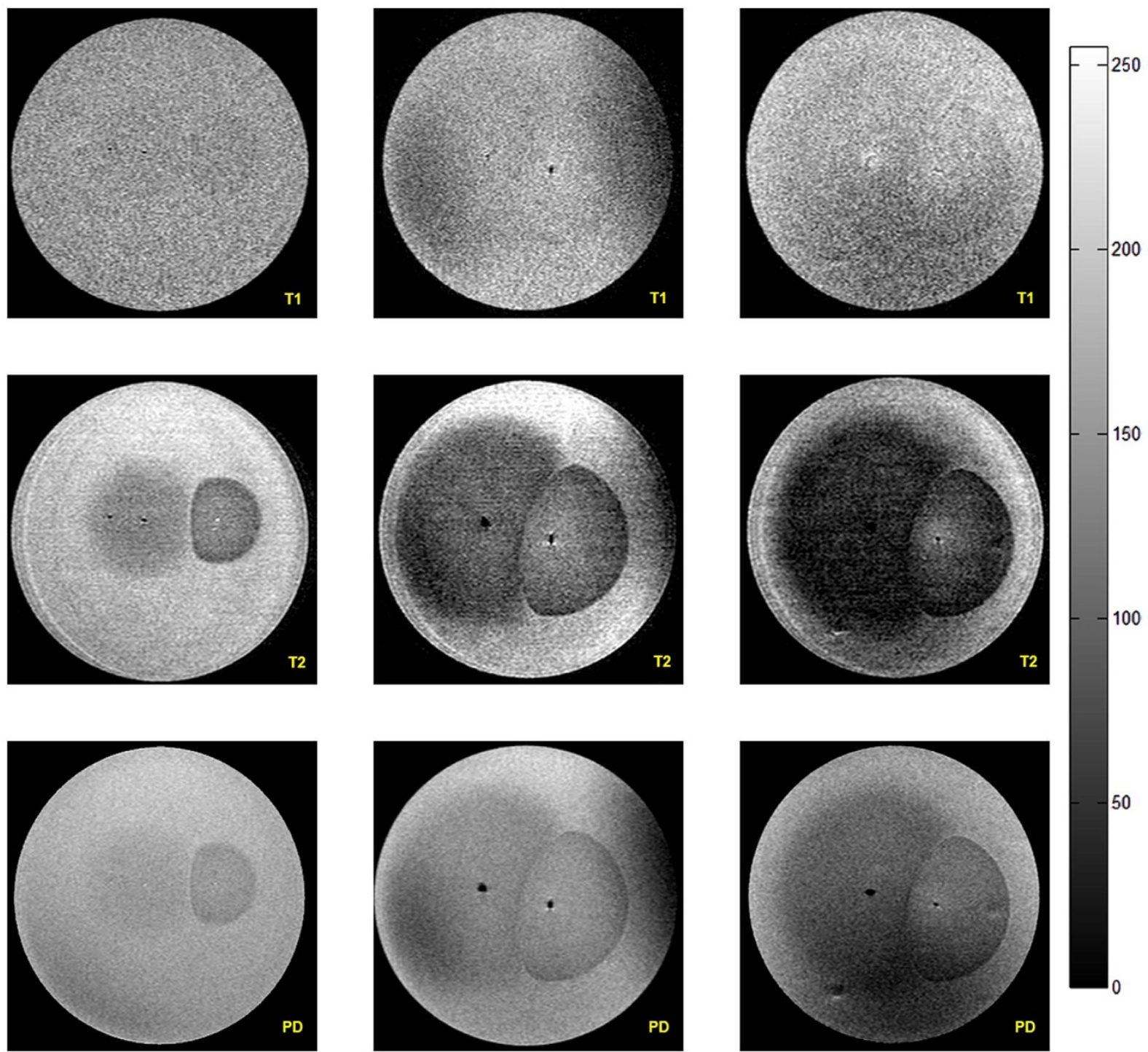

50

Figure 1 Comparative MRI imaging results. Each row corresponds to a sequence modality (Top to bottom: T1, T2, PD). Each column corresponds to a stimulation voltage (Left to right: $3 \mathrm{~V}, 6 \mathrm{~V}, 9 \mathrm{~V}$ ).

collides with the anode produced front at a line between the electrode and cathode. The outer margin of the lesion that has a circular shape is most likely at a $\mathrm{pH}$ of 8.2 , while the central line could be at any $\mathrm{pH}$ in the range of $\mathrm{pH} 8.2$ to $\mathrm{pH} \mathrm{12}$. It is interesting to note that immediately near the electrode for the $9 \mathrm{~V}$ voltage the intensity of the image is reduced compared to a region further away from the electrode.

The second row shows the results of $\mathrm{pH}$ staining using the Hagen wide range $\mathrm{pH}$ testing kit. The cathodic region on the left is marked with a distinct blue color which indicates a basic $\mathrm{pH}$ in the vicinity of 8.3 , while the anodic region on the right is marked with pink color which corresponds to $\mathrm{pH}$ level of 6.4. We have used the color-matching card provided by the manufacturer to establish the $\mathrm{pH}$ ranges. For $3 \mathrm{~V}$, the $\mathrm{pH}$ change affected regions have a circular shape. Increasing the voltage increases the size of the affected region. Just as for the other $\mathrm{pH}$ dye, and MRI images, the larger voltages lead to colliding $\mathrm{pH}$ fronts, which can observed as a straight line. Several interesting phenomena can be seen in the Hagen stained samples. First, for voltages of $6 \mathrm{~V}$ and $9 \mathrm{~V}$, the areas immediately adjacent to the electrodes appear discolored relative to the surrounding stained areas. Furthermore, on the cathode side at $6 \mathrm{~V}$ and $9 \mathrm{~V}$ there is a drop of fluid, which was observed on the top of the gel. For $9 \mathrm{~V}$, some of the dye has leaked into this drop and stained it.
pH probe experiments. To correlate the $\mathrm{pH}$ data captured by MRI and the $\mathrm{pH}$ sensitive dyes, the $\mathrm{pH}$ measurements made by the $\mathrm{pH}$ probe are presented in Table 2 and visualized graphically in the fifth row of Figure 4. The discrete $\mathrm{pH}$ measurements are presented on a single chart for each voltage. The effect of growing voltage is clearly seen: the extreme value of $\mathrm{pH}$ reached on both fronts is more deviant from the baseline normal $\mathrm{pH}$. The data is presented in two forms, with the measurements made from left to right and from right to left. The precision of the $\mathrm{pH}$ reading is 0.1 and the precision of the data point location is $+/-0.5 \mathrm{~mm}$. The difference between the left to right measurement curve and the right to left measurement curve is indicative of the systematic error of this measurement.

Bacterial Viability Experiment. To demonstrate the relevance of our work to a biological model, electrolytic stimulation was applied to an agar dish plated with $E$. Coli bacteria. The third row in figure 3 shows optical images of a bacterial viability pattern after treatment with $3 \mathrm{~V}$, $6 \mathrm{~V}$ and $9 \mathrm{~V}$ for 15 minutes, captured using a digital camera after 24 hour growth period. The anodic region on the right is marked with a clear bactericidal region increasing in area with increasing voltage. The cathodic region on the left is significantly smaller in terms of bactericidal area and is barely observable in the $3 \mathrm{~V}$ image. 

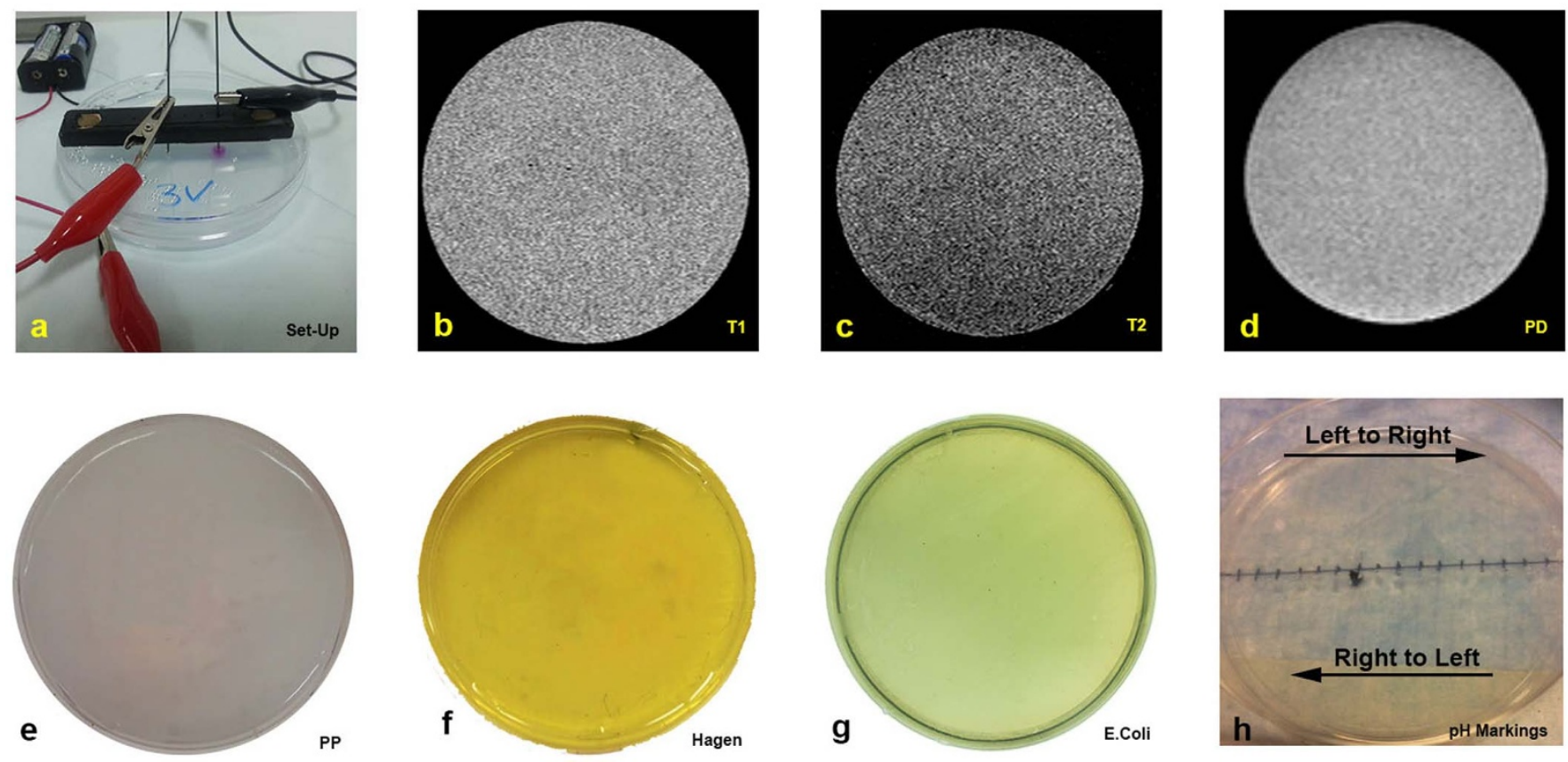

Figure 2 Experimental setup and control images for different experimental modalities used in this work. (a) Experimental setup, (b) T1 control study, (c) T2 control study, (d) PD control study (e) Phenolphtalein 1\%, (f) Hagen wide range pH indicator dye, (g) E. Coli control study.

\section{Discussion}

As mentioned in the introduction, our hypothesis that MRI can be used to image the $\mathrm{pH}$ fronts during electrolysis is based on reports in refs 30 and 38. As can be observed in the T1W images (Figure 1 first row), the treated volume exhibits hypointense to isointense signal, which indicates that the effect of electrolysis is minimal on T1W signal. A T1-weighted sequence produces an image where the signal contrast is determined by the differences in $\mathrm{T} 1$ relaxation times. The tissue signal in a $\mathrm{T} 1$ weighted imaging mode is inversely proportional to its T1 relaxation time. A short echo time (TE) is used to minimize T2-weighting together with a short repetition time (TR). A T1weighted image is typically characterized by dark fluid signal due to the long T1 relaxation time of water. This result is consistent with the original findings of Meiboom, Luz and Gill who studied proton relaxation times in water as a function of $\mathrm{pH}^{30}$ and show that $\mathrm{T} 1$ in water does not change in the range of from $\mathrm{pH} 2$ to $\mathrm{pH} 12$,

Visible changes are produced by electrolysis in T2 weighted images (Figure 1, second row). In the T2-weighted imaging mode, the signal contrast is determined by differences in $\mathrm{T} 2$ relaxation times. The tissue signal in a T2 weighted image is proportional to its T2 relaxation time. A long repetition time (TR) is used to minimize T1-weighting together with a long echo time. The results in figure 1 are also consistent with the original findings of Meiboom, Luz and Gill ${ }^{30}$. Their findings show that T2 in water is strongly affected by changes in $\mathrm{pH}$ and it increases symmetrically around $\mathrm{pH} 7$ with an increase and decrease in $\mathrm{pH}$. Shilling et al. ${ }^{38}$ provide a mechanistic explanation for their observed changes in T2 with changes in $\mathrm{pH}$ in the brain which is consistent with the findings of Meiboom et. al. by noting that "The effect of $\mathrm{pH}$ on spin-spin relaxation time (T2) might be explained by the fact that at $\mathrm{pH} 7.0$, i.e., in the neutral environment, water molecules build larger hydrogenbound mediated clusters than in the acid or base ranges. The reduced mobility leads to a prolonged correlation time for the dipolar interactions, which leads to a shortening of T2"39. Figure 1 shows that the electrolysis affected region near the anode is larger than that near the cathode. This difference makes physical sense and can be attributed to the relative radii of protons $\left(\mathrm{H}^{+}, 0.88 \mathrm{fm}\right)$ and hydroxide ions $\left(\mathrm{OH}^{-}, 110 \mathrm{pm}\right)$. Due to their relative smaller size, the protons are more mobile hence contributing to a larger extent to the conductivity increase around the anode. The increased mobility causes the $\mathrm{pH}$ region around the anode to be larger than around the cathode.

Proton Density (PD) is defined as the number of proton spins per unit volume of a tissue. Proton density may differ from the true water content due to short T2 components, which are not seen in MRI. So $\mathrm{PD}$-weighted imaging where the T1 and T2 effects are minimized leads to images whose contrast is determined primarily by the spin (proton) density. This requires a short TE and long TR. In figure 1, the third row shows the process of electrolysis generated PD MRI images, which correspond well with the T2W images. This confirms that the observed images are related to the electrolysis caused diffusion of protons and hydroxide ions.

In figure 3, the first and the second rows show the results of the process of electrolysis obtained with $\mathrm{pH}$ stained dyes. While the optical $\mathrm{pH}$ results cannot be quantitatively compared with the MR images, because the $\mathrm{pH}$ dyes have a restricted range, both the MRI and $\mathrm{pH}$ dyes images show similar phenomena and trends. The observed affected zone increase in both modalities with an increase in voltage, which is consistent with the increased production of electrolytic compounds with voltage. The anode and cathode electrolysis affected regions meet at the same location in both the $\mathrm{pH}$ dye images and the MRI images. The $\mathrm{pH}$ dye results, in particular the second row of figure 3, show some additional interesting physical phenomena. The effect relates to the observed drops of water on the surface of the gel, during electrolysis with $6 \mathrm{~V}$ and $9 \mathrm{~V}$. It is known that in an electric field, water moves by electro-osmosis from the anode to the cathode 6 . Therefore, during electrolysis, the gel near the anode tends to dehydrate while water accumulates near the cathode. This is the source of the water observed in the second row of figure 3 . We tentatively suggest that this electro-osmotic migration of water is responsible for the discoloration adjacent to the cathode observed with both MRI and $\mathrm{pH}$ dyes.

In figure 3, row three shows viability results from electrolysis treated E. Coli, grown on the surface of the gel. This part of the work is clinically relevant because electrolysis is becoming an important method for sterilizing surfaces and wounds, considering the growing antibiotic resistances of microorganisms ${ }^{40}$. The pattern of cell ablation observed here is consistent with electrolytic ablation and further supports the idea that the MRI detected changes are relevant to 

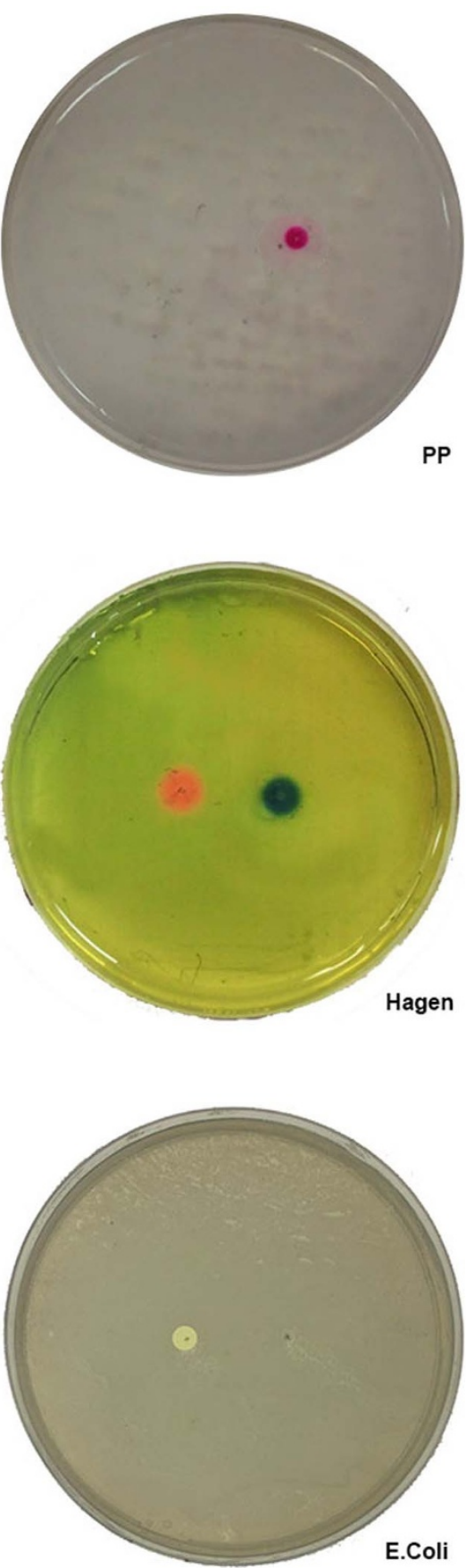
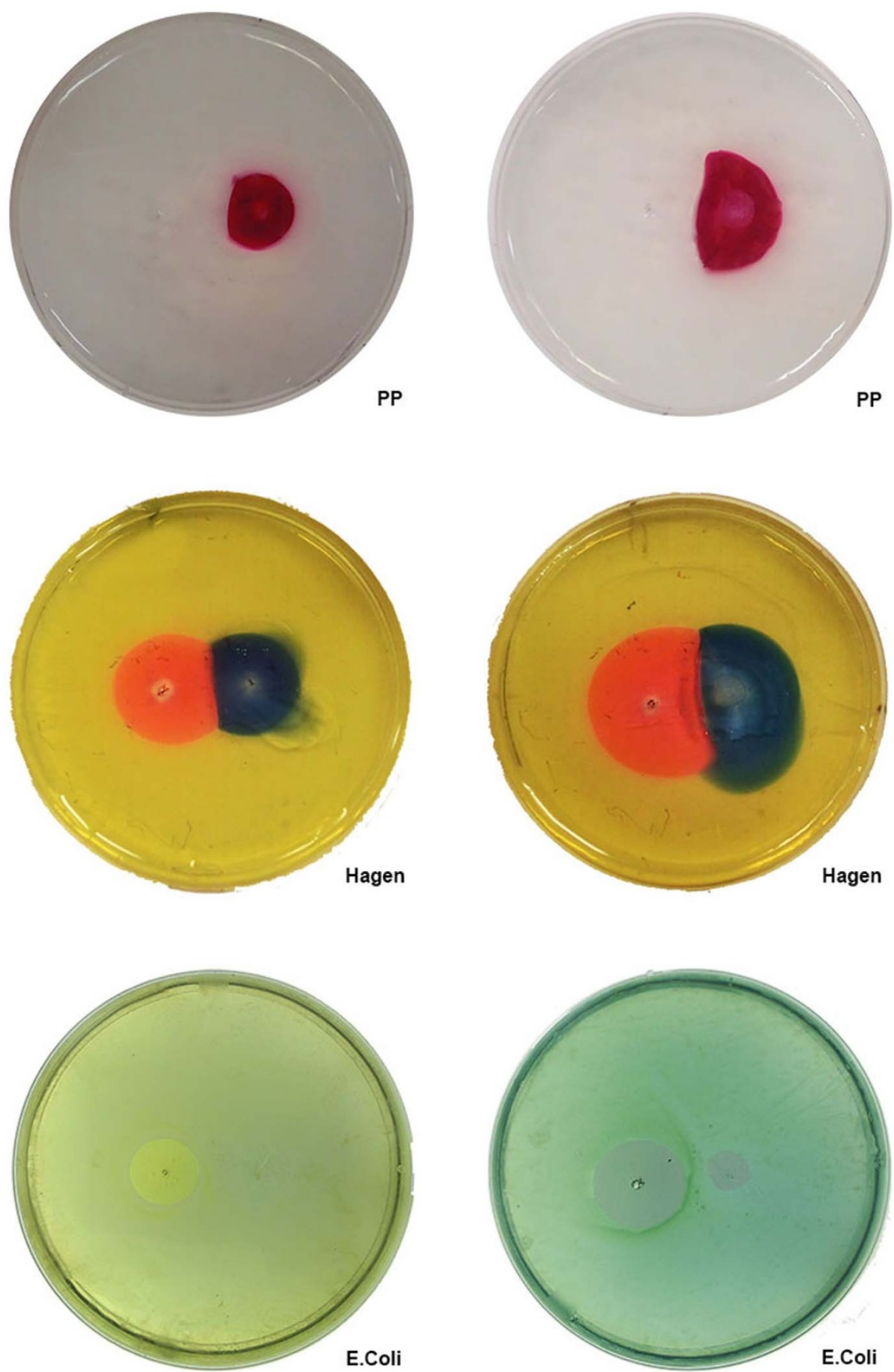

Figure 3 Comparative pH dyes and bacterial viability results. Each row corresponds to a control modality (Top to bottom: Phenolphtalein $1 \%$ pH indicator,Hagen $\mathrm{pH}$ indicator, E. Coli bacterial viability). Each column corresponds to a stimulation voltage (Left to right: $3 \mathrm{~V}, 6 \mathrm{~V}, 9 \mathrm{~V}$ ).

electrolysis. Figure 3, row three shows that the extent of cell ablation increases with the voltage and charge delivered as expected from a $\mathrm{pH}$ ablation process driven by electrolysis. It is also well established that the electrolytic products of the anode are more effective at cell ablation than the products of the cathode ${ }^{6}$. This is also confirmed in this study, which shows a much larger ablation zone near the anode than near the cathode.

In Figure 4, a comparison between MRI images, $\mathrm{pH}$ dye based images, discrete $\mathrm{pH}$ measurements and bacterial viability data shows that all the experiments, produce qualitatively similar results with respect to the effect of voltage on the affected area and with respect to the difference between the anodic and cathodic regions. A quantitative comparison is not possible because the $\mathrm{pH}$ dyes and the bacterial viability images represent limited ranges of $\mathrm{pH}$, while the discrete $\mathrm{pH}$ measurements suffer from systematic errors. However, the results from the different imaging techniques show that increasing the voltage (charge delivered) increases the affected area in both the anode and cathode affected volume, the anodic front advances faster than the cathodic front, and the anode and cathode affected regions meet on the line perpendicular to the line connecting the electrodes. Local $\mathrm{pH}$ measurements presented in the fifth row of Figure 4 are consistent qualitatively with the other means of $\mathrm{pH}$ monitoring, as they indicate more extreme $\mathrm{pH}$ ranges for higher voltages.

Figure 4 was brought here to summarize the results. The first row shows the T2W MR image onto which we have superimposed the outline of the $\mathrm{pH}$ dye image (rows two and three) and the outline of the viability experiment (row four). It is interesting to notice that the interface between the anode and the cathode affected zones lie on the 


\begin{tabular}{|c|c|c|c|c|c|c|}
\hline Distance from edge[mm] & $3 \vee R L$ & $3 \vee L R$ & $6 \mathrm{VRL}$ & $6 V L R$ & $9 \vee R L$ & $9 \mathrm{VLR}$ \\
\hline 0 & 7.94 & 7.7 & 9.05 & 7.35 & 9.08 & 7.15 \\
\hline 0.5 & 7.89 & 7.55 & 8.98 & 7.09 & 8.98 & 6.57 \\
\hline 1 & 7.86 & 7.25 & 7.92 & 7.15 & 8.46 & 6.7 \\
\hline 1.5 & 7.83 & 7.32 & 7.06 & 3.98 & 8.06 & 3.35 \\
\hline 2 & 7.55 & 7.3 & 6.3 & 2.47 & 7.82 & 2.53 \\
\hline 2.5 & 6.55 & 4.6 & 5.47 & 1.92 & 7.8 & 2.19 \\
\hline 3 & 6.43 & 3.97 & 5.29 & 1.98 & 8.15 & 1.87 \\
\hline 3.5 & 8.72 & 5.87 & 5.4 & 1.93 & 11.45 & 1.81 \\
\hline 4 & 9.19 & 6.67 & 10.6 & 10.25 & 12.52 & 11.53 \\
\hline 4.5 & 9.84 & 7.59 & 12.17 & 11.7 & 12.45 & 11.63 \\
\hline 5 & 9.95 & 9.96 & 12.15 & 11.84 & 12.46 & 11.63 \\
\hline 5.5 & 8.10 & 9.02 & 11.98 & 11.73 & 12.31 & 11.57 \\
\hline 6 & 8.01 & 7.81 & 11.15 & 11.36 & 11.58 & 11.29 \\
\hline 6.5 & 7.88 & 7.63 & 8.1 & 11.02 & 9.91 & 9.57 \\
\hline 7 & 7.8 & 7.26 & 8.07 & 9.65 & 9.41 & 7.95 \\
\hline 7.5 & 7.6 & 7.1 & 8.00 & 9.3 & 8.7 & 7.71 \\
\hline 8 & 7.35 & 7.04 & 7.97 & 9.34 & $\mathrm{n} / \mathrm{a}$ & $\mathrm{n} / \mathrm{a}$ \\
\hline
\end{tabular}

same line in the MRI image and the $\mathrm{pH}$ dye image - suggesting that they both represent the same phenomenon. The overall shape of the $\mathrm{pH}$ dye image is similar to the MRI image. The affected zone observed with MRI is larger than that observed with dyes, because the range of changes that can be observed with MRI is not restricted by a certain $\mathrm{pH}$ dye value. The extent of cell ablation is substantially less than the extent of the region in which MRI detects changes in $\mathrm{pH}$. In the past, studies on the effect of electrolysis on cell death were carried out using $\mathrm{pH}$ probes or $\mathrm{pH}$ dyes. This study suggests that MRI could become a useful tool in fundamental research on the effect of electrolysis on cells.

To address the temporal resolution of the MRI based approach and its relevance in the context of a typical length of an electrolysis procedure, we'd like to note that the resolution is limited by the time it takes to run a single scan which is given by $N \cdot N_{p} \cdot T_{r}$ where $N$ represents the number of signals averaged (NSA excitations), $N_{p}$ represents the size of the encoding matrix and $T_{r}$ represents the time to repetition. When multiple echoes are acquired after each excitation, this allows for accelerating the scan time and occasionally is referred to as the Turbo Spin Echo (TSE) or Turbo Factor. In such a case, the time given above should by divided by the Turbo Factor. For a typical choice of parameters, e.g. in our case study, the scan time falls in the range of 2-4 minutes. This is shorter than a typical length of an electrolytic treatment procedure and is much shorter than the time it would take to generate a complete scan with discrete $\mathrm{pH}$ measurements. This further suggests that MRI could be a potential method to study electrolysis in tissue.

\section{Methods}

Experimental Outline. The experiment was designed to allow for a comparison between different images of $\mathrm{pH}$ fronts produced by the electrolysis of a physiological saline solution phantom. The images were generated by various MRI sequences and compared with: a) optical images acquired using $\mathrm{pH}$-sensitive dyes embedded in a physiological saline agar solution phantom treated with electrolysis and b) bacterial $E$. Coli model, grown on a phantom and treated by applying the same electrolysis protocol. Each experimental study was done separately. In addition we have also collected a set of discrete measurements using a micro $\mathrm{pH}$ probe.

Tissue model. Our tissue model consists of a physiological saline based agar gel phantom with electrical conductivity designed to simulate that of a tissue. To construct the phantom, 1\% Bacto-Agar (Fisher Scientific) was mixed with $0.9 \mathrm{~g} / \mathrm{l}$ Sodium Chloride (Fisher Scientific) in distilled water. The solution was then brought to a boil and poured into $85 \mathrm{~mm}$ diameter Petri dishes. The same dish dimension was used in all the studies. The conductivity of the agar phantom was measured to be approximately $0.14 \mathrm{~S} / \mathrm{m}$ which is close to the range of hepatic tumor conductivity ${ }^{41}$.

Experimental procedure. The experimental setup is shown in Figure 2.a. We have used two disposable graphite electrodes made of pencil lead (Pentel super HB
$0.7 \mathrm{~mm}$ ), to avoid contamination with products of electrolysis from the electrodes. The electrodes, mounted in a horizontal holder were placed perpendicularly to the gel phantom in the Petri dish. The electrodes were inserted $7 \mathrm{~mm}$ deep into the gel at a distance of $2 \mathrm{~cm}$ between their centers. The electrodes are connected to constant voltage batteries. We used $3 \mathrm{~V}, 6 \mathrm{~V}$ and $9 \mathrm{~V}$ batteries. The electrolysis process lasted 15 minutes. While typical electrolysis stimulation is administered using a fixed current source, we have used a fixed voltage source and have taken current measurements during the procedure (data not shown) for charge dosage estimation purposes. The overall delivered charge dosages over the 15 minutes stimulation period were approximately $0.9 \mathrm{C}, 1.8 \mathrm{C}$ and $2.9 \mathrm{C}$, for $3 \mathrm{~V}, 6 \mathrm{~V}$ and $9 \mathrm{~V}$, respectively. These charge dosages fall within the range of a typical electrolytic ablation therapy stimulation $^{3,42}$. Identical experiments were done separately for MRI imaging, $\mathrm{pH}$ dyes based optical imaging, discrete $\mathrm{pH}$ measurements and the E. Coli viability model.

MRI Experimental Model. The phantom models were scanned, before and after electrolysis in a clinical 1.5 T MRI system (Philips Achieva SE) using a SENSE pediatric coil. The specific MRI parameters of each sequence are listed in Table 1. The mean acquisition time for each sequence was 3 minutes. Images shown in the paper were taken in the following order: T1W, T2W and PD. For later comparison with the MR images produced after electrolysis, figures 2.b, 2.c and 2.d show the baseline images for the sequences introduced above, respectively. In repeated experiments (results not shown) we changed the order and the time after the end of electrolysis at which the various sequences were taken and found no measurable effect of the time at which the images were acquired on the dimensions of the affected region.

pH-Sensitive Dye Model. As a control study, we used 2 distinct, $\mathrm{pH}$ sensitive dyes in order to estimate the boundary of the region where the $\mathrm{pH}$ has changed due to electrolysis. The dyes employed were 1) Phenolphtalein $1 \%$ by Sigma (turns pink in $\mathrm{pH}$ range of 8.2-12) and 2) Nutrafin $\mathrm{pH}$ wide range by Hagen (indicates $\mathrm{pH}$ by color in the range of 4.5-9). The dye manufacturer instructions prescribed the concentration of the last dye. We used a digital camera (Casio Exilim EX-ZR100) to acquire optical images of the experimental chamber before and after electrolysis and correlate these images with the images acquired using MRI and bacterial viability. For later comparison with images produced after electrolysis, figures 2.e and 2.f, show the baseline images prior to electrolysis for the two dyes used, respectively.

Discrete measurements with micro $\mathbf{p H}$ probes. To produce numerical values for $\mathrm{pH}$ we have used a MI-4146 Micro-combination $\mathrm{pH}$ probe (Microelectrodes, Inc. 40 Harvey Rd., Bedford, NH) with a response time of 5 seconds to 15 seconds in a stirred solution and a pH meter pH150 Oakton (625 East Bunker Court., Vernon Hills, IL). The experimental procedure is similar to that used in the MRI study and the $\mathrm{pH}$ sensitive dye study. We have marked a line through the diameter of the Petri dish and marked off every $5 \mathrm{~mm}$ (see Fig $1 . \mathrm{h}$ ). The two electrodes were placed at a distance of $2 \mathrm{~cm}$ between them. Similarly to the previous experiments we applied voltages of $3 \mathrm{~V}$, $6 \mathrm{~V}$ and $9 \mathrm{~V}$ for 15 minutes. After the electro-stimulation, the electrodes were removed, and $\mathrm{pH}$ measurements were made, starting with the marking furthest away from the dish center. To minimize and evaluate the effect of diffusion on the systematic errors of this method, in one set of the experiments the reading was performed from left to right, and in another set of experiments the reading was performed from right to left. The $\mathrm{pH}$ probe was placed over the mark and into the gel, making certain that the tip was covered. We waited until the reading was stabilized, usually between 15 and 25 seconds. The reading was recorded and the $\mathrm{pH}$ probe flushed and moved over to the next mark until the entire dish was transversed. One transverse reading took about 20 to 25 minutes. (No new electrolytic products were produced during the measurement period, however the $\mathrm{pH}$ changed during the 
$3 v$
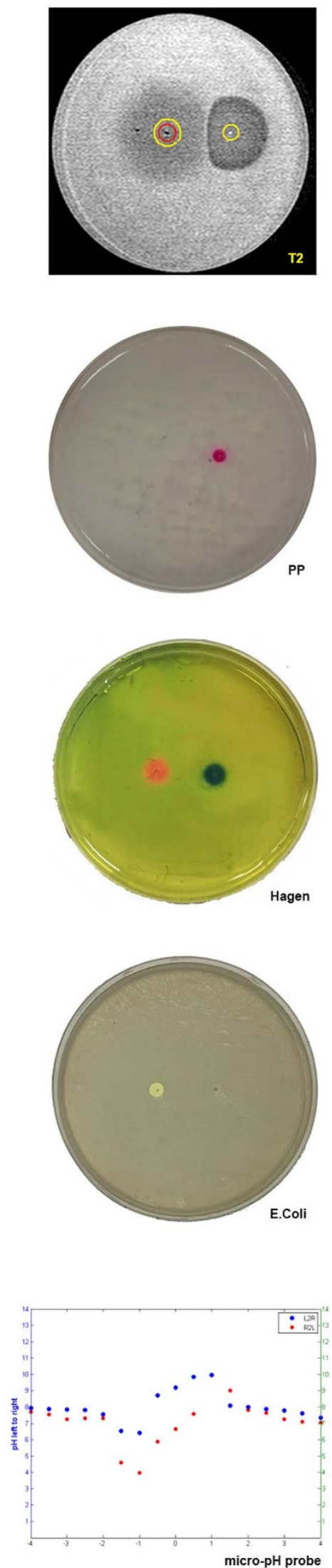

$6 v$
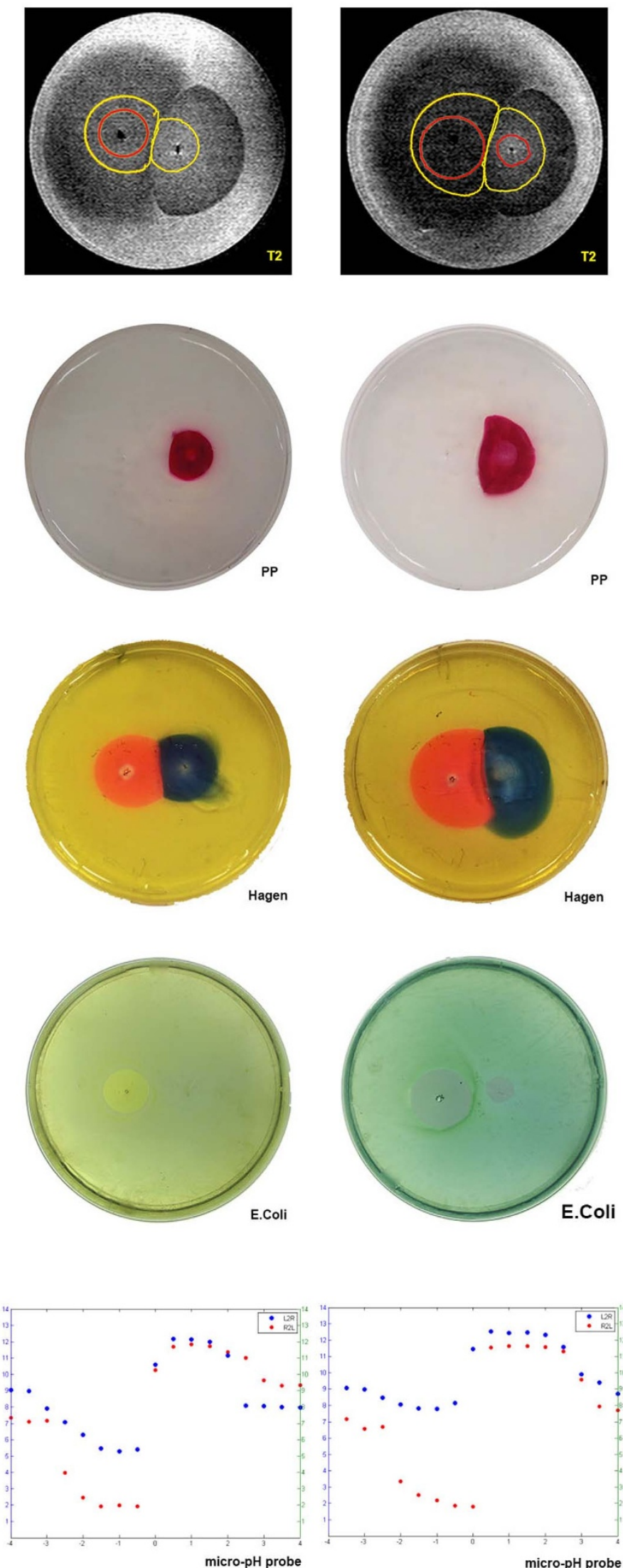

$9 v$
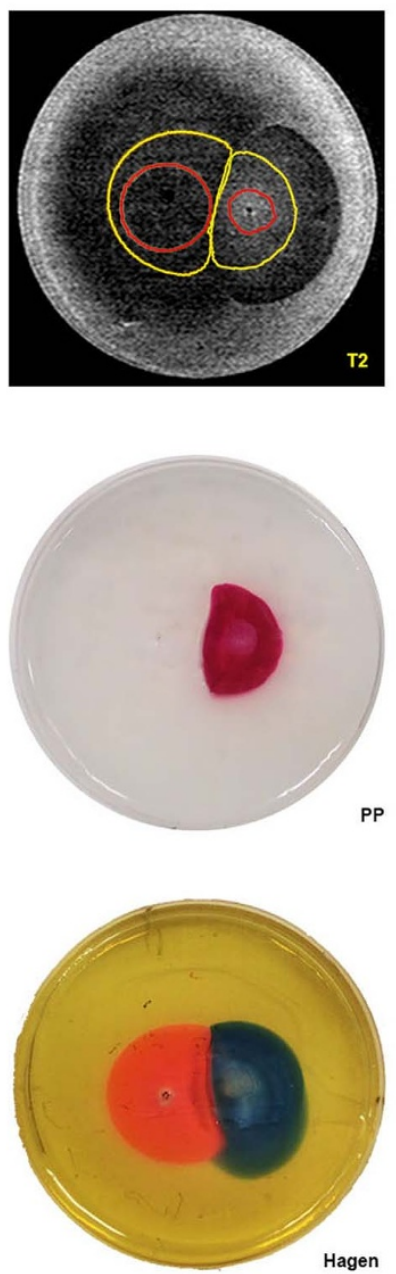

Hagen

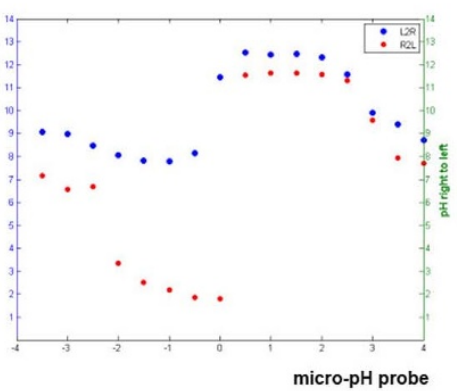

Figure 4 Comparative $\mathrm{pH}$ dyes and bacterial viability results. Each row corresponds to a control modality (Top to bottom: Phenolphtalein $1 \% \mathrm{pH}$ indicator,Hagen $\mathrm{pH}$ indicator, E. Coli bacterial viability, Local $\mathrm{pH}$ measurements taken by $\mathrm{pH}$ probe). Each column corresponds to a stimulation voltage (Left to right: $3 \mathrm{~V}, 6 \mathrm{~V}, 9 \mathrm{~V}$ ). 
measurements because of diffusion). While the actual data recorded is with a precision of 0.1 , the accuracy in relation to the colorimetric dye and MRI measurements is low. Therefore, the discrete $\mathrm{pH}$ measurement should be viewed as a rough indication of the actual values. We also considered measuring $\mathrm{pH}$ during the electro-stimulation process, however, we found, in other studies, that the insertion of an active $\mathrm{pH}$ probe during electrolysis distorts the reading because of the effect of the current on the $\mathrm{pH}$ probe.

Bacterial Model. Lyophilized E. Coli of HB101 strain (BioRad) were grown in LB broth overnight and plated on LB broth based agar gel filled petri dishes. The LB broth for the overnight growth consisted of 1\% BactoTryptone (BD), 0.5\% Yeast Extract (BD), $1 \% \mathrm{NaCl}$ (Sigma Aldrich) and 1.5\% Agarose (Sigma Aldrich). $6 \mathrm{~mm}$ glass beads (Sigma Aldrich) were used for plating to ensure uniform coverage. After plating, the beads were removed and the plates were incubated for 15 minutes at $37^{\circ}$. The conductivity of the gel was measured around $0.2 \mathrm{~S} / \mathrm{m}$. After electrolysis the Petri dishes were covered and incubated for 24 hours. We used a digital camera (Casio Exilim EX-ZR100) to acquire optical images of the areas where bacterial growth was inhibited and correlated these images with the images acquired using MRI and gels with $\mathrm{pH}$ dyes. For later comparison with images produced after electrolysis, figure 2.g shows the baseline images for an untreated with electrolysis, cell growth plate.

\section{Conclusion}

In summary, we report experimental findings that support the hypothesis that electrolysis induced $\mathrm{pH}$-changes can be detected with MRI. Our results indicate the feasibility of using MRI as a means to monitor dynamic changes in local $\mathrm{pH}$ level of a biological sample during an electrolysis process. Our work uses an agar-based gel model with conductivity in the range of a biological tissue, and is validated vs. optical images utilizing $\mathrm{pH}$ indicator dyes. In addition we demonstrate the relevance of our work in the biological context by correlating bacterial viability data with MRI measurements. It may be interesting to work on developing different MRI techniques for detecting $\mathrm{pH}$, using MRI markers. Reference ${ }^{30}$, suggests that it may be also possible to develop MRI sequences that detect discretely various ranges of $\mathrm{pH}$, because $\mathrm{T} 2$ seems to be very sensitive to $\mathrm{pH}$. Our results are promising, and invite further experimental explorations on the use of MRI for studying electrolysis and in clinical practice.

1. Amory, R. A treatise on electrolysis and its therapeutical and surgical treatement in disease. (William Woof \& Co., 1886).

2. Nordenstrom, B. E. W. Preliminary clinical trials of electrophoretic ionization in the treatement of malignant tumors. IRCS Medical Sc. 6, 537 (1978).

3. Nordenstrom, B. E. Electrochemical treatment of cancer. I: Variable response to anodic and cathodic fields. Am J Clin Oncol 12, 530-536 (1989).

4. Nilsson, E., Berendson, J. \& Fontes, E. Development of a dosage method for electrochemical treatment of tumours: a simplified mathematical model. Bioelectroch Bioener 47, 11-18, doi:10.1016/s0302-4598(98)00157-3 (1998).

5. Nilsson, E., Berendson, J. \& Fontes, E. Electrochemical treatment of tumours: a simplified mathematical model. J Electroanal Chem 460, 88-99, doi:10.1016/ s0022-0728(98)00352-0 (1999).

6. Nilsson, E. et al. Electrochemical treatment of tumours. Bioelectrochemistry $\mathbf{5 1}$ 1-11, doi:10.1016/s0302-4598(99)00073-2 (2000).

7. von Euler, H., Nilsson, E., Olsson, J. M. \& Lagerstedt, A. S. Electrochemical treatment (EchT) effects in rat mammary and liver tissue. In vivo optimizing of a dose-planning model for EChT of tumours. Bioelectrochemistry 54, 117-124, doi:10.1016/s1567-5394(01)00118-9 (2001)

8. von Euler, H., Nilsson, E., Lagerstedt, A. S. \& Olsson, J. M. Development of a doseplanning method for electrochemical treatment of tumors: A study of mammary tissue in healthy female CD rats. Electro Magnetobiol 18, 93-+ doi:10.3109/ 15368379909012903 (1999).

9. Bergues Pupo, A. E., Bory Reyes, J., Bergues Cabrales, L. E. \& Bergues Cabrales, J. M. Analytical and numerical solutions of the potential and electric field generated by different electrode arrays in a tumor tissue under electrotherapy. Biomed Eng Online 10 doi:10.1186/1475-925x-10-85 (2011).

10. Placeres Jimenez, R. et al. 3D Stationary Electric Current Density in a Spherical Tumor Treated With Low Direct Current: An Analytical Solution. Bioelectromagnetics 32, 120-130, doi:10.1002/bem.20611 (2011).

11. Turjanski, P. et al. $\mathrm{pH}$ front tracking in the electrochemical treatment (EChT) of tumors: Experiments and simulations. Electrochimica Acta 54, 6199-6206, doi:10.1016/j.electacta.2009.05.062 (2009).

12. Camue Ciria, H. M. et al. Antitumor effects of electrochemical treatment. Chn J Cancer Res 25, 223-234, doi:10.3978/j.issn.1000-9604.2013.03.03 (2013).

13. Yoon, D.-S. et al. Introduction of electrochemical therapy (EChT) and application of EChT to the breast tumor. J. Breast Canc 10, 162-168 (2007).
14. Czymek, R. et al. Electrochemical Treatment: An Investigation of Dose-Response Relationships Using an Isolated Liver Perfusion Model. Saudi J Gastro 17, 335-342, doi:10.4103/1319-3767.84491 (2011).

15. Griffin, D., Dodd, N., Zhao, S., Pullan, B. \& Moore, J. V. Low-level direct electrical current therapy for hepatic metastases. I. Preclinical studies on normal liver. Brit J Cancer 72, 31-34 (1995).

16. Griffin, D., Dodd, N. J., Moore, J. V., Pullan, B. \& Taylor, T. The effects of low-level direct current therapy on a preclinical mammary carcinoma: tumour regression and systemic biochemical sequelae. Brit J Cancer 69, 875-878 (1994).

17. Miklavčič, D. et al. Tumor treatment by direct electric current-tumor temperature and $\mathrm{pH}$, electrode material and configuration. Bioelectrochem Bioenerget 30, 209-220 (1993).

18. Miklavčič, D., Fajgelj, A. \& Serša, G. Tumor treatment by direct electric current: electrode material deposition. Bioelectrochem Bioenerget 35, 93-97 (1994).

19. Finch, J. G. et al. Liver electrolysis: $\mathrm{pH}$ can reliably monitor the extent of hepatic ablation in pigs. Clin Sci 102, 389-395 (2002).

20. Olaiz, N., Suarez, C., Risk, M., Molina, F. \& Marshall, G. Tracking protein electrodenaturation fronts in the electrochemical treatment of tumors. Electrochem Commun 12, 94-97, doi:10.1016/j.elecom.2009.10.044 (2010).

21. Ivic, M. L. A., Perovic, S. D., Zivkovic, P. M., Nikolic, N. D. \& Popov, K. I. An electrochemical illustration of the mathematical modelling of chlorine impact and acidification in electrochemical tumour treatment and its application on an agaragar gel system. J Electroanal Chem 549, 129-135, doi:10.1016/s00220728(03)00251-1 (2003).

22. Moore, E. W. Determination of $\mathrm{pH}$ by the glass electrode: $\mathrm{pH}$ meter calibration for gastric analysis. Gastroenterology 54, 501-507 (1968).

23. Takmakov, P. et al. Characterization of local pH changes in brain using fast-scan cyclic voltammetry with carbon microelectrodes. Anal Chem 82, 9892-9900 (2010).

24. Venton, B. J., Michael, D. J. \& Wightman, R. M. Correlation of local changes in extracellular oxygen and $\mathrm{pH}$ that accompany dopaminergic terminal activity in the rat caudate-putamen. J Neurochem 84, 373-381 (2003).

25. De Beer, D., Schramm, A., Santegoeds, C. M. \& Kuhl, M. A nitrite microsensor for profiling environmental biofilms. Appl Environ Microbiol 63, 973-977 (1997).

26. Chu, K. F. \& Dupuy, D. E. Thermal ablation of tumours: biological mechanisms and avdances in therapy. Nat Rev Cancer 14, 199-208 (2014).

27. Turjanski, P. et al. $\mathrm{pH}$ front tracking in the electrochemical treatment (EChT) of tumors: Experiments and simulations. Electrochimica Acta 54, 6199-6206 (2009).

28. Turjanski, P. et al. The role of $\mathrm{pH}$ fronts in reversible electroporation. PLoS One 6, e17303 (2011).

29. Maglietti, F. et al. The Role of Ph Fronts in Tissue Electroporation Based Treatments. PLoS One 8, e80167 (2013).

30. Meiboom, S., Luz, Z. \& Gill, D. Proton Relaxation In Water. J Chem Phys 27, 1411-1412, doi:10.1063/1.1744015 (1957).

31. Kettunen, M. I., Grohn, O. H. J., Silvennoinen, M. J., Penttonen, M. \& Kauppinen, R. A. Effects of intracellular $\mathrm{pH}$, blood, and tissue oxygen tension on T-1 rho relaxation in rat brain. Magn Reson Med 48, 470-477, doi:10.1002/mrm.10233 (2002).

32. McVicar, N. et al. Quantitative tissue $\mathrm{pH}$ measurement during cerebral ischemia using amine and amide concentration-independent detection (AACID) with MRI. J Cerebr Blood F Met 34, 690-698, doi:10.1038/jcbfm.2014.12 (2014).

33. Zong, X., Wang, P., Kim, S. G. \& Jin, T. Sensitivity and Source of Amine-Proton Exchange and Amide-Proton Transfer Magnetic Resonance Imaging in Cerebral Ischemia. Magn Reson Med 71, 118-132 (2014).

34. Longo, D. L., Busato, A., Lanzardo, S., Antico, F. \& Aime, S. Imaging the pH evolution of an acute kidney injury model by means of iopamidol, a MRI-CEST pH-responsive contrast agent. Magn Reson Med 70, 859-864, doi:10.1002/ mrm.24513 (2013).

35. Evans, S. \& Hall, L. Evaluation of a range of MRI-active $\mathrm{pH}$ indicators using a multiple-sample method. Aiche Journal 51, 1541-1547, doi:10.1002/aic.10387 (2005).

36. Evans, S. \& Hall, L. Measurement of $\mathrm{pH}$ in food systems by magnetic resonance imaging. Can J Chem Eng 83, 73-77 (2005).

37. Schmid, A. I. et al. Exercising calf muscle T-2 changes correlate with $\mathrm{pH}, \mathrm{PCr}$ recovery and maximum oxidative phosphorylation. Nmr Biomed 27, 553-560, doi:10.1002/nbm.3092 (2014).

38. Schilling, A. M., Blankenburg, F. B., Bernarding, J., Heidenreich, J. O. \& Wolf, K. J. Intracerebral $\mathrm{pH}$ affects the T2 relaxation time of brain tissue. Neuroradiology 44, 968-972, doi:10.1007/s00234-002-0873-0 (2002).

39. Schilling, A., Blankenburg, F., Bernarding, J., Heidenreich, J. \& Wolf, K. Intracerebral $\mathrm{pH}$ affects the $\mathrm{T} 2$ relaxation time of brain tissue. Neuroradiology 44 , 968-972 (2002).

40. Piggott, J. M., Berney, H., Hurley, E. \& Clair, J. Planar flexible electrode for use in wound sterilization. Paper presented at 24th Annual International Conference of the Engineering in Medicine and Biology Society. Annual Fall Meeting of the Biomedical Engineering Society, 1929-1930 vol. 1923, doi:10.1109/ iembs.2002.1053098 (2002).

41. Laufer, S., Ivorra, A., Reuter, V. E., Rubinsky, B. \& Solomon, S. B. Electrical impedance characterization of normal and cancerous human hepatic tissue. Physiol Meas 31, 995 (2010). 
42. Nordenström, B. Biologically closed electric circuits: Clinical, experimental and theoretical evidence for an additional circulatory system. (Ursus Medical AB, 1983).

\section{Author contributions}

A.M. has performed the bacterial and ph-dye experiments, prepared figures 3-4 and wrote the paper. M.H. has performed the MRI experiments, prepared table 1, figures 1-2 and wrote the paper. L.R. prepared table 2 and performed the micro-pH experiments. B.R. has conceived the experiments and contributed to writing the paper.

\section{Additional information}

Competing financial interests: The authors declare no competing financial interests.

How to cite this article: Meir, A., Hjouj, M., Rubinsky, L. \& Rubinsky, B. Magnetic Resonance Imaging of Electrolysis.. Sci. Rep. 5, 8095; DOI:10.1038/srep08095 (2015).

This work is licensed under a Creative Commons Attribution 4.0 International License. The images or other third party material in this article are included in the article's Creative Commons license, unless indicated otherwise in the credit line; if the material is not included under the Creative Commons license, users will need to obtain permission from the license holder in order to reproduce the material. To view a copy of this license, visit http://creativecommons.org/licenses/by/4.0/ 